\title{
Discovery of the INTEGRAL X/ $\gamma$-ray transient IGR J00291+5934: A Comptonised accreting ms pulsar?
}

\author{
S. E. Shaw ${ }^{1,2}$, N. Mowlavi ${ }^{2,4}$, J. Rodriguez ${ }^{3,2}$, P. Ubertini ${ }^{5}$, F. Capitanio ${ }^{5}$, K. Ebisawa ${ }^{6,2}$, D. Eckert ${ }^{2,4}$, \\ T. J.-L. Courvoisier ${ }^{2,4}$, N. Produit ${ }^{2,4}$, R. Walter ${ }^{2,4}$, and M. Falanga ${ }^{3}$ \\ 1 School of Physics and Astronomy, University of Southampton, SO17 1BJ, UK \\ 2 INTEGRAL Science Data Centre, 1290 Versoix, Switzerland \\ e-mail: simon.shaw@obs .unige.ch \\ 3 CEA Saclay, DSM/DAPNIA/SAp (CNRS FRE 2591), 91191 Gif-sur-Yvette Cedex, France \\ 4 Observatoire de Genève, 51 Chemin des Maillettes, 1290 Sauverny, Switzerland \\ 5 Istituto di Astrofisica Spaziale, CNR/INAF, via Fosso del Cavaliere 100, 00133 Rome, Italy \\ ${ }^{6}$ NASA Goddard Space Flight Center, Code 661, Building 2, Greenbelt, MD 20771, USA
}

Received 16 December 2004 / Accepted 23 January 2005

\begin{abstract}
We report the discovery of a high-energy transient with the IBIS/ISGRI detector on board the INTEGRAL observatory. The source, namely IGR J00291+5934, was first detected on 2nd December 2004 in the routine monitoring of the IBIS/ISGRI 20-60 keV images. The observations were conducted during Galactic Plane Scans, which are a key part of the INTEGRAL Core Programme observations. After verifying the basic source behaviour, the discovery was announced on 3rd December. The transient shows a hard Comptonised spectrum, with peak energy release at about $20 \mathrm{keV}$ and a total luminosity of $\sim 0.9 \times 10^{36} \mathrm{erg} \mathrm{s}^{-1}$ in the $5-100 \mathrm{keV}$ range, assuming a distance of $3 \mathrm{kpc}$. Following the INTEGRAL announcement of the discovery of IGR J00291+5934, a number of observations were made by other instruments. We summarise the results of those observations and, together with the INTEGRAL data, identifiy IGR J00291+5934 as the 6th member of a class of accreting $\mathrm{X}$-ray millisecond pulsars.
\end{abstract}

Key words. gamma-rays: observations - pulsars: individual IGR J00291+5934

\section{Introduction}

IGR J00291+5934 was discovered on 2nd December 2004 (Eckert et al. 2004), during the routine monitoring of IBIS/ISGRI 20-60 keV images of Galactic Plane Scan (GPS) observations at the INTEGRAL Science Data Centre (ISDC). In following GPS observations, on 8th December, the source flux remained basically stable at $\sim 8 \times 10^{-10} \mathrm{erg} \mathrm{cm}^{-2} \mathrm{~s}^{-1}$ with a marginal monotonic decrease. However, by 11th December, the source flux had reduced by around $50 \%$ (see Sect. 2).

The day after the discovery the same sky region was observed by the Rossi X-ray Timing Explorer (RXTE), which detected a $35 \mathrm{mCrab}$ excess with a coherent pulsation at $\sim 598.88 \mathrm{~Hz}(1.67 \mathrm{~ms})$ and pulsed fraction $\sim 6 \%$, making IGR J00291+5934 the fastest known accreting X-ray pulsar (Markwardt et al. 2004b). Further analysis of RXTE/Proportional Counter Array data, showed that the source has an orbital period of $147.412 \pm 0.006$ min (Markwardt et al. 2004a). The RXTE spectrum was consistent with an absorbed power law with an equivalent absorption column density $N_{\mathrm{H}} \sim 7 \times 10^{21} \mathrm{~cm}^{-2}$, and a photon index of $\sim 1.7$ (Markwardt et al. 2004b). Archival RXTE/All Sky Monitor data suggested that the source had also entered in outburst in 1998 and 2001, which may indicate that IGR J00291+5934 has a $\sim 3$ year recurrence time (Remillard 2004). No such activity was seen in archival BeppoSAX and INTEGRAL data (e.g. in 't Zand \& Heise 2004), although these instruments did not make contemporaneous observations with RXTE. Later observations by the Chandra X-ray telescope made a more accurate determination of $N_{\mathrm{H}}=(2.8 \pm 0.4) \times 10^{21} \mathrm{~cm}^{-2}$ (Nowak et al. 2004).

Observations at radio and optical wavelengths revealed the presence of a transient counterpart at a position consistent with that of the high-energy source, with possible optical emission features (Markwardt et al. 2004b; Pooley 2004; Roelofs et al. 2004; Fender et al. 2004). The most accurate optical position has been reported by Fox \& Kulkarni (2004), $(\alpha, \delta)=\left(00^{\mathrm{h}} 29^{\mathrm{m}} 03^{\mathrm{s}} .06,+59^{\circ} 34^{\prime} 19^{\prime} \cdot 0\right) \pm 00^{\prime} 5$; the source is located in the galactic plane, away from the galactic centre at $(l, b)=\left(120^{\circ} 0964,-3 \circ 1765\right)$.

In view of the high-energy behaviour, the presence of pulsations, the short orbital period and other similarities with the object SAX J1808.4-3658 (in 't Zand et al. 1998; Wijnands \& van der Klis 1998; in't Zand et al. 2001) we consider IGR J00291+5934 to be the 6th member of a class of accreting $\mathrm{X}$-ray binaries with weakly magnetised pulsars. 


\subsection{INTEGRAL}

The INTEGRAL satellite was launched on 17 October 2002 and contains several instruments dedicated to observing the high-energy sky in the $3 \mathrm{keV}-10 \mathrm{MeV}$ band (Winkler et al. 2003). The prime instruments for this work are the following: the coded mask imager IBIS/ISGRI, which is sensitive in the $15 \mathrm{keV}-1 \mathrm{MeV}$ band and has a large $29^{\circ} \times 29^{\circ}$ field of view (Lebrun et al. 2003; Ubertini et al. 2003); the X-ray monitor JEM-X, which is sensitive from 3-30 keV and has a 13.2 diameter field of view (although noise towards the rim of the detector limits the usable area for weak sources to the central 10.5 ; Lund et al. 2003). The JEM-X instrument consists of two identical telescopes, but for this work, only the JEM-X1 unit was operational.

Data from the satellite are analysed very quickly after an observation; the ISDC Quick Look Analysis (QLA) pipeline runs continuously on the incoming telemetry (Courvoisier et al. 2003). Images are produced by QLA in the following energy bands: 3-10 keV and 10-30 keV (JEM-X); 20-60 keV and 60-200 keV (ISGRI). The images are automatically monitored for new or highly variable sources, which can lead to an automatic alert being issued with a delay of $<2 \mathrm{~h}$ from the end of the observation. All images are also inspected manually by the ISDC Scientist on Duty (Shaw et al. 2004).

In this INTEGRAL observing period, $\sim 30 \%$ of the total amount of observing time is split between the Galactic Centre Deep Exposure (GCDE) and the GPS. The GPS are regular pointings in a saw-tooth pattern along the Galactic Plane, between galactic latitude $b= \pm 10^{\circ}$, conducted every $\sim 12$ days (one INTEGRAL revolution is 3 days long). Each GPS pointing, or Science Window (ScW), lasts $2200 \mathrm{~s}$ and is separated from the next one by $6^{\circ}$ (Winkler et al. 2003).

\section{Discovery of IGR J00291+5934}

The ISDC QLA pipeline first suggested that a new $\mathrm{X} / \gamma$-ray source had been discovered in ISGRI images of GPS observations, by an automatic alert issued on 2nd December 2004 at 09:00:19 UTC (Eckert et al. 2004). The alert was triggered because a previously unknown source was detected, at a significance $>10 \sigma$, in a $20-60 \mathrm{keV}$ ISGRI image of GPS pointing 0261-2 (ScW 2 of INTEGRAL revolution 0261). This was confirmed at 09:42:03 UTC after the following pointing, 0261-3, by another alert issued at the same sky position. In both cases the alerts were issued approximately $1 \mathrm{~h}$ and $40 \mathrm{~min}$ after the end of the pointing (see Table 1 for a summary).

Further QLA images showed that the source persisted in the ISGRI QLA images in 0261-3, and had also been detected in the JEM-X1 instrument in 0261-2 (albeit at a level below that required to trigger an automatic alert). Due to the progression of the GPS, the source was not in the instrument field of view for the following pointings. The pointing 0261-2 was also the first pointing of the revolution that could be analysed, since the previous pointing was affected by the passage of the satellite through the Earth's radiation belts. The next GPS observations, during revolution 0263 on 8th December 2004, also yielded four detections of the source by ISGRI. However, in the
Table 1. Detections of IGR J00291+5934 by the ISDC QLA pipeline, based on ISGRI data from single GPS pointings during INTEGRAL revolutions 0261 (2nd December 2004), 0263 (8th December 2004) and 0264 (11th December 2004). The detected position, angular distance of the source from the spacecraft pointing axis $(\theta)$ and 20-60 keV count-rate $(F)$ are shown. The last line shows the detection by JEM-X1 and the $3-10 \mathrm{keV}$ flux. Pointings marked with * are those where the source was not automatically detected and localised manually.

\begin{tabular}{lcccc}
\hline \hline $\begin{array}{l}\text { Pointing } \\
(\text { Rev-ScW })\end{array}$ & $\begin{array}{c}\text { UTC Start } \\
(\mathrm{hh}: \mathrm{mm})\end{array}$ & $\begin{array}{c}(\alpha, \delta) \\
\left({ }^{\circ}\right)\end{array}$ & $\begin{array}{c}\theta \\
\left({ }^{\circ}\right)\end{array}$ & $\begin{array}{c}F_{20-60} \\
(\mathrm{cps})\end{array}$ \\
\hline $0261-2$ & $06: 43$ & $(7.26,59.57)$ & 3.3 & $7.5 \pm 0.5$ \\
$0261-3$ & $07: 23$ & $(7.24,59.58)$ & 6.7 & $6.1 \pm 0.5$ \\
$0261-4^{*}$ & $08: 03$ & $(7.3,59.57)$ & 12.2 & $7.9 \pm 1.2$ \\
$0263-1^{*}$ & $06: 31$ & $(7.3,59.57)$ & 10.2 & $5.5 \pm 0.8$ \\
$0263-2$ & $07: 10$ & $(7.28,59.58)$ & 6.7 & $4.3 \pm 0.5$ \\
$0263-3$ & $07: 50$ & $(7.27,59.58)$ & 7.6 & $4.6 \pm 0.6$ \\
$0263-4^{*}$ & $08: 30$ & $(7.3,59.57)$ & 11.8 & $5.3 \pm 1.0$ \\
$0264-2^{*}$ & $06: 18$ & $(7.28,59.59)$ & 10.4 & $1.9 \pm 0.8$ \\
$0264-3 *$ & $06: 58$ & $(7.4,59.5)$ & 4.6 & $2.2 \pm 0.5$ \\
$0264-4^{*}$ & $07: 38$ & $(7.29,59.55)$ & 2.3 & $2.6 \pm 0.5$ \\
$0264-5 *$ & $08: 18$ & $(7.2,59.5)$ & 7.9 & $2.4 \pm 0.7$ \\
\hline $0261-2$ & $06: 43$ & $(7.279,59.568)$ & 3.3 & $1.6 \pm 0.2$ \\
\hline
\end{tabular}

following GPS pointing, conducted on 11th December (revolution 0264), the source was observed to have faded in ISGRI, and not detected at all in JEM-X.

After the detection, the source was also observed in an already scheduled observation of the CasA/Tycho region and was the subject of an INTEGRAL ToO observation, which began on 6th December. The analysis of these data is the responsibility of the respective PIs, and will not be discussed here (Falanga et al. 2005, in preparation).

\subsection{Analysis of INTEGRAL GPS observations}

The ISGRI GPS pointings, listed in Table 1, have been analysed using the standard OSA 4.2 software $^{1}$. Figure 1 shows the location of the source in an ISGRI mosaic image, made from all 7 pointings of revolutions 0261 and 0263 , and the single JEM-X detection. In both instruments the source is very clearly identified with the optical position of Fox \& Kulkarni (2004). Within the ISGRI mosaic image the HMXB 3A 0114+650 is also detected; this gives confidence that some problem with the spacecraft pointing is not responsible for falsely identifying a new source and that IGR J00291+5934 is not a misidentification of another nearby object.

The most significant detection of IGR J00291+5934 was during the first pointing, 0261-2, and it was possible to construct a composite JEM-X1 and ISGRI spectrum. However, the sensitivity of ISGRI is such that it is hard to constrain a physical fit to this source on the strength of just one pointing. A simple absorbed power-law, with the value of $N_{\mathrm{H}}=0.2 \times 10^{22} \mathrm{~cm}^{-2}$

1 The OSA software can be obtained from www.isdc. unige.ch 

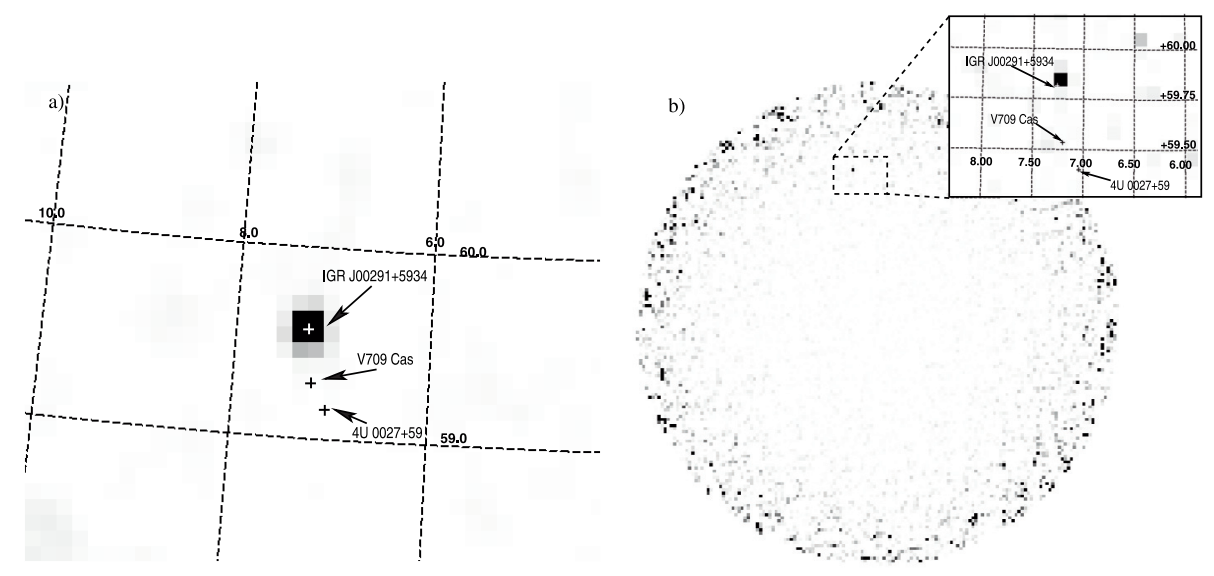

Fig. 1. a) Zoomed 15-40 keV ISGRI significance image made from a mosaic of 7 exposures from revolutions 0261 and 0263 (see Table 1 ). b) 3-10 keV JEM-X1 intensity image, with zoomed inset corresponding to the dashed area (diameter of main image is 10.5). In both images a squared grey-scale, ranging from 0 to the peak pixel value (23 $\sigma$ for ISGRI, $4.4 \mathrm{cps}$ for JEM-X1) has been used. It is clear that the INTEGRAL detections are co-located with the optical position of IGR J00291+5934 reported by Fox \& Kulkarni (2004) (white cross) and not with the positions of other nearby sources.

fixed, gives a reduced $\chi_{v}^{2}=1.1$. The photon index is $1.81 \pm 0.13$ corresponding to a 5-50 keV flux of $8.3_{-2.6}^{+3.7} \times 10^{-10} \mathrm{erg} \mathrm{cm}^{-2} \mathrm{~s}^{-1}$ in agreement with Markwardt et al. (2004b).

The broad band count-rates of IGR J00291+5934 are also noted in Table 1 . These show that the flux faded slightly during the course of $\sim 10$ days, as reported by Swank \& Markwardt (2004). Note that the uncertainties on flux measurements with ISGRI increases with the off-axis angle, $\theta$, as the source flux is not fully coded by the mask.

If IGR J00291+5934 is an accreting ms pulsar, then a power-law does not necessarily describe the physics of the source and it is interesting to investigate the possibility of a Comptonised spectrum. To increase the high-energy statistics, an average ISGRI spectrum was made, using the method described in Rodriguez et al. (2005), from those pointings in Table 1 with $\theta<10^{\circ}$; this was added to the JEM-X1 spectrum from 0261-2. A reasonable fit was obtained with the CompST model (Sunyaev \& Titarchuk 1980): $\chi_{v}^{2}=0.7$, electron temperature $k T=25_{-7}^{+21} \mathrm{keV}$ and optical depth $\tau=3.6_{-1.3}^{+1.0}$; the $5-100 \mathrm{keV}$ flux is $8.5_{-0.2}^{+0.5} \times 10^{-10} \mathrm{erg} \mathrm{cm}^{-2} \mathrm{~s}^{-1}$. However, it should be noted that a simple power-law also gives a valid fit, $\chi_{v}^{2}=1.0$, albeit with a softer photon index of $2.05 \pm 0.10$. The drop in the flux above $100 \mathrm{keV}$ confirms the presence of a cutoff in the spectrum at high energies (Fig. 2).

Unfortunately, the source was not bright enough to extend the high-energy spectral information with the INTEGRAL spectrometer (SPI; Vedrenne et al. 2003). The source was, however, detected at a corresponding position to the other instruments, $(\alpha, \delta)=\left(6.3,+59^{\circ} .8\right) \pm 1^{\circ} .0$, and the measured $20-60 \mathrm{keV}$ flux of $(5.3 \pm 1.6) \times 10^{-10} \mathrm{erg} \mathrm{cm}^{-2} \mathrm{~s}^{-1}$ (assuming a photon index of 1.8) is in good agreement with ISGRI.

The ISGRI data have also been searched for pulsations at the $1.67 \mathrm{~ms}$ period of Markwardt et al. (2004b). To maximize the signal to noise ratio, the $20-60 \mathrm{keV}$ band was used, noisy pixels were removed and only those pixels that were fully illuminated by IGR J00291+5934 were selected. The event arrival time was corrected to the solar barycentre

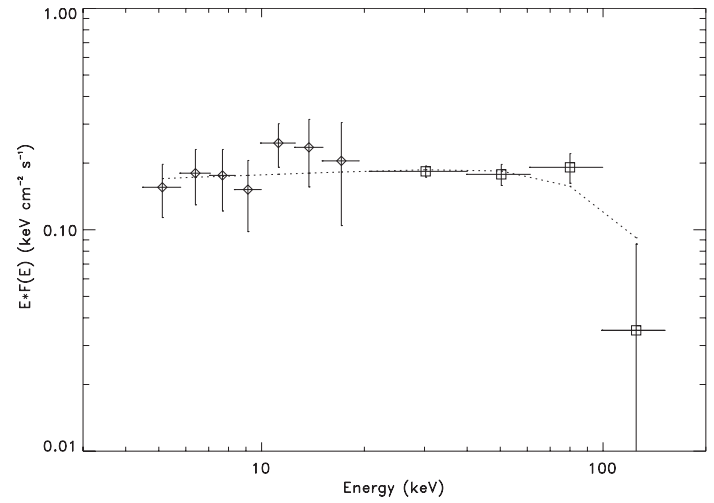

Fig. 2. X/ $\gamma$-ray spectrum of IGR J00291+5934 made with JEM-X1 from observation 0261-2 (circles), and an average of ISGRI observations (squares, see text for data selection). The line shows a simultaneous fit to the two sets of data with the CompST model.

and a folded analysis was conducted around the known pulsefrequency of $598.88 \mathrm{~Hz}$. An upper limit for the pulsed amplitude at $598.88 \mathrm{~Hz}$ was found to be $\sim 20 \%$, which is consistent with the value of $6 \%$ by Markwardt et al. (2004b).

\section{Discussion}

We report the discovery of the fastest known accreting X-ray pulsar, IGR J00291+5934, with INTEGRAL. It is likely that IGR J00291+5934 is a low mass X-ray binary (LMXB) system containing a NS pulsar that has been spun up by accretion of material, from a companion star, via an accretion disc. IGR J00291+5934 is one of the fastest X-ray pulsars discovered to date and is second only to PSR B1937+21 (an isolated pulsar showing radio and X-ray pulsations at $P \sim 1.57 \mathrm{~ms}$; Backer et al. 1982; Takahashi et al. 2001). The absorbing column measured by Nowak et al. (2004) with Chandra is approximately a factor of two lower than the estimate of the galactic total on the same line of sight $\left(N_{\mathrm{H}} \sim 4.5 \times 10^{21} \mathrm{~cm}^{-2}\right.$; Dickey \& Lockman 1990). Given that the source is $120^{\circ}$ from 
the Galactic Centre and assuming that the galactic disk has a radius of $13 \mathrm{kpc}$, with the Earth $8.5 \mathrm{kpc}$ from the centre then the average density of absorbing material in the source direction is $\sim 0.2 \mathrm{~cm}^{-3}$. Assuming no local absorption puts an upper limit on the source distance of $\sim 3.3 \mathrm{kpc}$. Although this is a highly simplistic argument, it seems likely that the source is reasonably local. It should also be noted that the Perseus arm of the Milky Way, at $l=120^{\circ}$, is located approximately $2.5 \mathrm{kpc}$ away (Taylor \& Cordes 1993). Using $3 \mathrm{kpc}$ as an estimate of the source distance, and the $5-100 \mathrm{keV}$ flux quoted in Sect. 2.1, gives a luminosity for the source of $\sim 0.9 \times 10^{36} \mathrm{erg} \mathrm{s}^{-1}$.

The other five known ms X-ray pulsars (SAX J1808.4-3658, XTE J1751-305, XTE J0929-314, XTE J1807-294, XTE J1814-338), are believed to be old NS (age 10 $0^{9}$ years), with moderately weak magnetic field, $B \sim 10^{8} \mathrm{G}$ (see e.g. Chakrabarty et al. 2003; Wijnands et al. 2003). In fact all of them are transient systems with short orbital periods, accreting at very low rates. This implies that the magnetic field of the NS is very weak, which is also suggested by the perceived age of the systems (Heindl \& Smith 1998; Titarchuk et al. 2002). The INTEGRAL high-energy spectral information is limited with this very small data set, but is consistent with properties of other ms X-ray pulsars, with $\tau \sim 3$ and $k_{\mathrm{B}} T \sim 20 \mathrm{keV}$. The upper limit on the high-energy pulsations, coupled with the peak energy release at $\sim 24 \mathrm{keV}$, could be consistent with a Comptonised flux being emitted from a hot plasma near the inner part of the disc. Some of this plasma may be channeled towards the NS by the magnetic field, resulting in the pulsed part of the spectrum. In this model, the seed photons would be supplied by the cold intermediate part of the disc rather than the higher temperature NS black-body emission.

It is remarkable that in all of these objects the pulse fraction is of the same order $(\sim 6 \%)$. IGR J00291+5934, shares many common characteristics with the other objects, particularly SAX J1808.4-3658. The latter has a relatively similar orbital period of $\sim 2 \mathrm{~h}$ (Chakrabarty \& Morgan 1998), a $\sim 2$ year recurrence time of the outburst and is also the only other known ms pulsar for which a radio counterpart was detected during outburst (Gaensler et al. 1999). It is reasonable to assume that IGR J00291+5934 is an old NS that has been spun up by the accretion of material, with a magnetic field of the same order as the other ms pulsars. The spectral analysis of IGR J00291+5934 reveals that this source is more similar to XTE J1814-338 than the 3 others for which evidence of black body radiation have been seen. However, this may simply be due to the high lower boundary of the JEM-X detector. This is reinforced by the fact that black body emission has been detected in SAX J1808.4-3658 with detectors allowing a broader coverage towards the low energies (e.g. Wijnands et al. 2003).

The spectral analysis of these objects reveal that they are not so different to other NS/pulsar LMXBs, in the sense that the emission processes are thought to originate through thermal + Comptonised processes. It is therefore quite puzzling that only some of these systems show persistent coherent pulsations at the NS spin period. The fact that all $\mathrm{ms}$ X-ray pulsars have very short orbital periods may be a clue to why these systems do or do not show persistent pulsations (see recent review by Wijnands 2005). Despite being the fastest accreting ms pulsar to date, it is interesting to note that the period remains significantly higher than $1 \mathrm{~ms}$.

Acknowledgements. This paper is based on observations made with the ESA INTEGRAL project. The authors thank A. Paizis for useful comments in the preparation of this paper. S.E.S. thanks PPARC for financial support. The useful comments and timely response of the anonymous referee were greatly appreciated.

\section{References}

Backer, D. C., Kulkarni, S. R., Heiles, C., Davis, M. M., \& Goss, W. M. 1982, Nature, 300, 615

Chakrabarty, D., \& Morgan, E. H. 1998, Nature, 394, 346

Chakrabarty, D., Morgan, E. H., Muno, M. P., et al. 2003, Nature, 424, 42

Courvoisier, T. J.-L., Walter, R., Beckmann, V., et al. 2003, A\&A, 411, L53

Dickey, J. M., \& Lockman, F. J. 1990, ARA\&A, 28, 215

Eckert, D., Walter, R., Kretschmar, P., et al. 2004, ATel, 352

Fender, R., de Bruyn, G., Pooley, G., \& Stappers, B. 2004, ATel, 361

Fox, D. B., \& Kulkarni, S. R. 2004, ATel, 354

Gaensler, B. M., Stappers, B. W., \& Getts, T. J. 1999, ApJ, 522, L117

Heindl, W. A., \& Smith, D. M. 1998, ApJ, 506, L35

in 't Zand, J. J. M., \& Heise, J. 2004, ATel, 362

in 't Zand, J. J. M., Heise, J., Muller, J. M., et al. 1998, A\&A, 331, L25

in 't Zand, J. J. M., Cornelisse, R., Kuulkers, E., et al. 2001, A\&A, 372,916

Lebrun, F., Leray, J. P., Lavocat, P., et al. 2003, A\&A, 411, L141

Lund, N., Budtz-Jørgensen, C., Westergaard, N. J., et al. 2003, A\&A, 411, L231

Markwardt, C. B., Galloway, D. K., Chakrabarty, D., \& Strohmayer, T. E. 2004a, ATel, 360

Markwardt, C. B., Swank, J. H., \& Strohmayer, T. E. 2004b, ATel, 353

Nowak, M. A., Paizis, A., Wilms, J., Ebisawa, K., et al. 2004, ATel, 369

Pooley, G. 2004, ATel, 355

Remillard, R. 2004, ATel, 357

Rodriguez, J., Cabanac, C., Hannikainen, D., et al. 2005, A\&A, in press [arXiv: astro-ph/0412555]

Roelofs, G., Jonker, P. G., Steeghs, D., Torres, M., \& Nelemans, G. 2004, ATel, 356

Shaw, S., Mowlavi, N., Ebisawa, K., et al. 2004, in 5th INTEGRAL Workshop - The INTEGRAL Universe: ESA SP-552, 897

Sunyaev, R. A., \& Titarchuk, L. G. 1980, A\&A, 86, 121

Swank, J. H., \& Markwardt, C. B. 2004, ATel, 365

Takahashi, M., Shibata, S., Torii, K., et al. 2001, ApJ, 554, 316

Taylor, J. H., \& Cordes, J. M. 1993, ApJ, 411, 674

Titarchuk, L., Cui, W., \& Wood, K. 2002, ApJ, 576, L49

Ubertini, P., Lebrun, F., Di Cocco, G., et al. 2003, A\&A, 411, L131

Vedrenne, G., Roques, J.-P., Schönfelder, V., et al. 2003, A\&A, 411, L63

Wijnands, R. 2005, in Pulsars New Research (NY: Nova Science Publishers), in press [arXiv: astro-ph/0501264]

Wijnands, R., \& van der Klis, M. 1998, Nature, 394, 344

Wijnands, R., van der Klis, M., Homan, J., et al. 2003, Nature, 424, 44 Winkler, C., Courvoisier, T. J.-L., Di Cocco, G., et al. 2003, A\&A, 411, L1 